\title{
CORRIGENDUM: ON MULTIPERIODIC WORDS
}

\author{
ŠTĚPÁn HOLUB ${ }^{1}$
}

\begin{abstract}
An algorithm is corrected here that was presented as Theorem 2 in [ک̌S. Holub, RAIRO-Theor. Inf. Appl. 40 (2006) 583-591]. It is designed to calculate the maximum length of a nontrivial word with a given set of periods.
\end{abstract}

Mathematics Subject Classification. 68R15.

The purpose of this contribution is to fill a gap in the algorithm presented in my paper [1] as Theorem 2. The theorem contains a formula that is supposed to yield the length $\mathcal{L}_{P}$ of the longest nontrivial multiperiodic word, that is, the longest word having a given set $P$ of coprime periods and not the period one. The formula reads as follows:

$$
\mathcal{L}_{P}=m_{n-1}-1+\sum_{i=0}^{n-1} m_{i},
$$

where $m_{i}$ is the minimal element of the set $Q_{i}$, which is given by the following recursive formula: $Q_{0}=P$, and

$$
Q_{i+1}=\left\{q-m_{i} \mid q \in Q_{i}, q \neq m_{i}\right\} \cup\left\{m_{i}\right\} .
$$

The number $n$ is established as the smallest index such that $1 \in Q_{n}$.

Gwénaël Richomme [2] pointed out that the formula is not correct, giving the following counterexample:

Consider the set $P=\{5,7,8\}$ of coprime periods. We have

$$
\begin{aligned}
& Q_{0}=\{5,7,8\}, \\
& Q_{1}=\{2,3,5\}, \\
& Q_{2}=\{1,2,3\} .
\end{aligned}
$$

Keywords and phrases. Periodicity, combinatorics on words.

1 Department of Algebra, Charles University, Sokolovská 83, 17586 Praha, Czech Republic. holub@karlin.mff.cuni.cz 
Therefore $n=2$, and

$$
m_{n-1}-1+\sum_{i=0}^{n-1} m_{i}=m_{1}-1+m_{0}+m_{1}=8 .
$$

However, the nontrivial word aaaabaaaa of length 9 has periods $P$.

The main idea of the proof of Theorem 2 is Lemma 2 claiming that (with an adjusted notation) for $k \geq m_{i}$, we have $\left[Q_{i}, k+m_{i}\right]=\left[Q_{i+1}, k\right]$. Recall that $[Q, \ell]$ denotes the maximum number of letters which can occur in a word of length $\ell$ having periods $Q$.

An additional observation is Lemma 3, according to which $[Q, 2 m-1]>1$ if $m=\min Q>1$.

To illustrate why Theorem 2 gives a wrong result for $P=\{5,7,8\}$ let us first look at an example where the formula works.

Let $P^{\prime}=\{3,5,8\}$, whence

$$
\begin{aligned}
& Q_{0}^{\prime}=\{3,5,8\}, \\
& Q_{1}^{\prime}=\{2,3,5\}, \\
& Q_{2}^{\prime}=\{1,2,3\} .
\end{aligned}
$$

We can now deduce, from Lemmas 2 and 3, that

$$
\left[Q_{0}^{\prime}, 6\right]=\left[Q_{1}^{\prime}, 3\right]>1
$$

while

$$
\left[Q_{0}^{\prime}, 7\right]=\left[Q_{1}^{\prime}, 4\right]=\left[Q_{2}^{\prime}, 2\right]=1
$$

Therefore $\mathcal{L}_{P}=6$.

Similar reasoning for $P=\{5,7,8\}$ would yield

$$
\left[Q_{0}, 8\right]=\left[Q_{1}, 3\right]>1
$$

and

$$
\left[Q_{0}, 9\right]=\left[Q_{1}, 4\right]=\left[Q_{2}, 2\right]=1 \text {, }
$$

leading to the wrong answer $\mathcal{L}_{P}=8$. The problem is that we cannot conclude $\left[Q_{0}, 9\right]=\left[Q_{1}, 4\right]$ due to the fact that the condition $k \geq m_{0}$ of Lemma 2 is not satisfied: we have $k=4$ and $m_{0}=5$. In fact, $\left[Q_{0}, 9\right] \neq\left[Q_{1}, 4\right]$ holds in this case. (Similarly, $\left[Q_{0}, 8\right] \neq\left[Q_{1}, 3\right]$ ).

This is precisely the situation that has to be taken into account in order to obtain a correct algorithm, which we state and proof now. To simplify notation, consider further only one step of the reduction and denote $P=Q_{0}, Q=Q_{1}$ and $m=\min P$ (this notation conforms to [1]). 
Theorem 1 (correction of Thm. 2 in [1]). Let $P \subset \mathbb{N}_{+}$be a set of positive integers such that $\operatorname{gcd}(P)=1$, and $m=\min (P)>1$. Let

$$
Q=\{q-m \mid q \in P, q \neq m\} \cup\{m\} .
$$

Then the maximal length of a nontrivial word with periods $P$ is given by the following recursive formula:

$$
\mathcal{L}_{P}=m+\max \left\{\mathcal{L}_{Q}, m-1\right\}
$$

where $\mathcal{L}_{Q}$ is the maximal length of a nontrivial word with periods $Q$, and is defined as 0 if $1 \in Q$.

Proof. As in [1], we can verify that for any $P$ (even infinite) the definition of $\mathcal{L}_{P}$ is correct, namely that the recursion terminates.

Let $\mathcal{L}_{Q} \geq m$. Lemma 2 now yields that $\left[P, \mathcal{L}_{Q}+m\right]=\left[Q, \mathcal{L}_{Q}\right] \neq 1$ while $\left[P, \mathcal{L}_{Q}+1+m\right]=\left[Q, \mathcal{L}_{Q}+1\right]=1$, which implies that $\mathcal{L}_{P}$ is equal to $\mathcal{L}_{Q}+m$.

Let $\mathcal{L}_{Q}<m$. Then $[Q, m]=1$ and Lemma 2 implies $[P, 2 m]=1$. The proof is concluded by Lemma 3 .

Note that the formula (1) is wrong if and only if $\mathcal{L}_{Q_{i+1}}<m_{i}-1$ for some $i<n-1$. The formula was formed under the (mistaken) assumption that this inequality holds only for $i=n-1$.

To conclude, let us apply the corrected theorem to the above counterexample $P=\{5,7,8\}$. We have

$$
\begin{aligned}
& \mathcal{L}_{Q_{2}}=0, \\
& \mathcal{L}_{Q_{1}}=2+\max \{0,1\}=3, \\
& \mathcal{L}_{Q_{0}}=5+\max \{3,4\}=9 .
\end{aligned}
$$

Acknowledgements. I am grateful to Gwénaël Richomme for finding the mistake and communicating it to me.

\section{REFERENCES}

[1] Š. Holub, On multiperiodic words. RAIRO-Theor. Inf. Appl. 40 (2006) 583-591.

[2] G. Richomme, personal communication (July 2011).

Communicated by A. Restivo.

Received August 3, 2011. Accepted October 13, 2011. 